\title{
Low-Complexity LDPC-Coded USTM Noncohereny MIMO Receivers
}

\author{
Li Peng, Lingling Yang \\ Wuhan National Laboratory for Optoelectronics, Department of Electronics and Information Engineering, \\ Huazhong University of Science and Technology, Wuhan, China \\ Email: pengli@mail.hust.edu.cn
}

Received 10 July 2014; revised 15 August 2014; accepted 20 September 2014

Copyright (C) 2014 by authors and OALib.

This work is licensed under the Creative Commons Attribution International License (CC BY). http://creativecommons.org/licenses/by/4.0/

(c) $\underset{E Y}{0}$ Open Access

\section{Abstract}

This paper proposes a scheme of combining low-density parity check (LDPC) code with unitary space time modulation (USTM) for noncoherent multiple-input-multiple-output (MIMO) transmitter and receiver over Rayleigh block fading and additive white Gaussian noise (AWGN) channel. The main aim is to design the low complexity coded noncoherent MIMO receiver which is completely dependent on the structural feature of unitary space-time matrix without sending the pilot symbol at the transmitter and estimating the channel state information at the receiver. Considering soft information required by belief-propagation (BP) iterative decoder of LDPC code, we deduce a maximum a posteriori probability (MAP) demodulating algorithm using a special USTM based on the sine-cosine function. A novel dual-demodulator is conceived for decreasing the computational complexity of this MAP demodulator. Furthermore, the iterative feedback scheme between MAP demodulator and BP decoder is introduced and its modified parameter scheme is considered for further improving performance of the dual-demodulator. Comparing with uncoded USTM, our LDPC-coded USTM MIMO receiver can obtain about $17 \mathrm{~dB}$ coding gain at $10^{-6} \mathrm{BER}$.

\section{Keywords}

Unitary Space-Time Modulation (USTM), Log-Likelihood Ratio (LLP), Maximum a Posteriori Probability (MAP), Demodulating Algorithm, Iterative Feedback, Low-Density Parity-Check (LDPC) Code

Subject Areas: Information and Communication Theory and Algorithms, Mobile and Portable Communications Systems

\section{Introduction}

Coherent multiple-input-multiple-output (MIMO) wireless communication system utilizing $M$ transmit and $N$ 
receive antennas in fading channels, which know the perfect channel state information (CSI) at the receiver, is appealing for its capacity of growing as $\min \{M, N\} \log (S N R)$ at high signal-to-noise ratios (SNRs) [1] [2], which provides higher data rate than single-input single-output (SISO) system. But the practical situation is that the perfect CSI may be unavailable at the receiver, which results in the difficulty of coherent demodulation. An alternative is noncoherent MIMO systems proposed by Hochwald et al. in [3] [4] which neither transmitter nor receiver knows CSI. Its capacity of a block-fading channel with coherent interval $T$ grows as

$M^{*}\left(1-M^{*} / T\right) \log (S N R)$ with $M^{*}=\min \{M, N, T / 2\}$ at high SNR [3] [5], which is $\left(1-M^{*} / T\right)$ times less than that of coherent MIMO system but it has potential practical demodulating scheme. In recent years, two studying directions of noncoherent MIMO modulating/demodulating system are widely investigated. One is that transmitters in [6]-[9] send both pilot symbol and data symbol in a coherent interval $T$ and receivers first estimate CSI by using the pilot symbol and then coherently demodulates the data information by means of the estimated CSI, which is called pilot symbol-assisted modulation (PSAM). The other is that transmitters send isotropically distribution unitary signals generated by unitary space time modulation (USTM) in [3] [4] and receives complete noncoherently demodulations based on the maximum likelihood (ML) by means of the structure features of an unitary space time matrix [10]-[12]. The drawback of the former is that sending pilot symbol at transmitter needs to consume channel bandwidth resource and channel estimation at receiver increases time-delay of system and leads to performance loss by channel estimation error. The drawback of the latter is that the ML demodulating algorithm based on unitary space-time matrix requires the calculation of constellation point traversal which leads to high complexity, especially for the large size of constellation.

At the present, many improved strategies of the above-mentioned drawbacks for two schemes are paid attention to in communication field. For examples, [7] [9] studied the schemes of information-bearing pilots which is likely to mitigate the bandwidth consumption of pilots, while [12] [13] involved the research of reducing the complexity of the ML algorithm based on USTM. As the drawback of time-delay formed by the channel estimation in MIMO systems with PSAM or information-bearing PSAM scheme is inevitable, so we focus on the design of the MIMO system based on complete unitary space-time matrix which need not estimate CSI. The key problem is to find the simple demodulating algorithm at receiver. It appears that few research findings of the simplified demodulating algorithm are published, but we can still catch a glimpse of two developing directions, such as constructing the special structural unitary matrix set which can generate simple demodulating algorithm [11] [12] and designing a general simplified demodulating algorithm for arbitrary unitary constellation [13].

The paper [11] provides a noncoherent unitary constellation $\left\{\boldsymbol{\Phi}_{l}\right\}_{l=0}^{L-1}$ in which the $l$ th unitary space time matrix has aspect $\Phi_{l}=\left[\sin (\pi l / L) \cdot \boldsymbol{I}_{M} \cos (\pi l / L) \cdot \boldsymbol{I}_{M}\right]_{T \times M}^{\dagger}$ for $l=0,1, \cdots, L-1$, where $L$ denotes the size of constellation and $\boldsymbol{I}_{M}$ is the $M \times M$ identity matrix, and we call it the USTM based on the sine-cosine function, the SC-USTM for short. Up to now, only SC-USTM can generate the simplest demodulation algorithm based on the maximum likelihood (ML) whose complexity is $O(M N)$ which is completely independent of the size of constellation $L$ and coherent interval $T$, unlike the other existing USTM schemes whose simple demodulation algorithms has $O(M N T \tilde{L})$ complexity, where $\tilde{L}<L$ denotes those constellation points which take part in the calculation after simplifying the ML demodulating algorithm [12] [13]. Because of this, this paper will use the SC-USTM to construct the coded USTM noncoherent MIMO system which is expected to achieve the lowest demodulating complexity under the condition of taking no account of CSI.

It is known from [3] that the capacity-achieving USTM is fit for the region of high SNR or when $T \gg M$. In order to make the MIMO system also achieve the channel capacity in the low-SNR region, as well as for noncoherent block fading channels with small $T$ values, it is widely acknowledged that channel coding should be considered in MIMO system. In recent few years, the noncoherent MIMO systems of combining error correcting codes with space-time modulation have been noted by the communication system designers. Multiple articles [7] [14]-[19] investigated the diversified concatenated schemes of combining Turbo code with USTM technique from different perspectives. In [16], USTM technique is introduced to the trellis code modulation (TCM) schemes. A convolutional coded sequence was first transformed into an interleaved sequence and then mapped the USTM signal matrix in [17]. The performance bound for the LDPC-coded USTM is investigated in [18], and in [6], a concatenated scheme of combining turbo-like code with pilot-assisted USTM was discussed.

The authors of this paper in [20] presented a concatenated scheme of combine an outer code of IRA-LDPC with an inner code of SC-USTM which needs not consider sending pilot symbols and estimating the CSI. This 
paper is a development of [20] through the following jobs. First, we deduce the simplifying MAP demodulating algorithm by using the structural feature of the SC-USTM based on the original MAP algorithm in [20], see Section III. For reducing the calculated amount of constellation point traversal in the MAP demodulating algorithm, we create the novel dual-demodulator which has nothing to do with the size of constellation $L$ and coherent interval $T$, and has only linear complexity in all antennas $M N$, see Section IV. In order to improve performance, we introduce iterative feedback mechanism between the MAP demodulator and the BP iterative decoder, and discuss the scheme of modifying the feedback parameter, in Section V. Section VI gives the analysis of complexity. Conclusions are drawn in Section VII.

\section{LDPC-CODED SC-USTM System Model}

Consider a channel model with $M$ transmit and $N$ receive antennas. The channels between transmit and receive antenna pairs are Rayleigh block fading channels and independent of each other. The fading coefficients remain constant for a block of $T$ symbol intervals and change independently from one block to another. Figure 1 gives the specific LDPC-coded SC-USTM system model operating on the above channel.

The transmitter contains two parts: LDPC encoder and SC-USTM modulator. An information sequence $\left(u_{0}, u_{1}, \cdots, u_{k-1}\right)$ is first encoded into a codeword sequence $\left(v_{0}, v_{1}, \cdots, v_{n_{c}-1}\right)$ by using the LDPC code with rate $R_{c}=k / n_{c}$, where $k$ and $n_{c}$ denote respectively the length of information bits and codeword. Taking in turn $a=\log _{2} L$ bits from the LDPC-coded sequence $\left(v_{0}, v_{1}, \cdots, v_{n_{c}-1}\right)$, we can construct the coded mapping sequence (CMS) $\mathbf{z}_{l}=\left(z_{0}, z_{1}, \cdots, z_{a-1}\right)$ for $l=0,1, \cdots, L-1$, where $L$ denotes the size of constellation. There are $2^{a}=L$ distinct CMSs which form a set $\left\{\mathbf{z}_{l}\right\}_{l=0}^{L-1}$.

We require that the transmit signal matrix (TSM) $\quad \boldsymbol{X}_{l}=\sqrt{T} \boldsymbol{\Phi}_{l}$ is designed as a $T \times M$ unitary matrix that satisfies $\boldsymbol{\Phi}_{l}^{\dagger} \boldsymbol{\Phi}_{l}=\boldsymbol{I}_{M}$ for $l=0,1, \cdots, L-1$, and $L$ transmit signal matrices form a unitary space-time constellation $\left\{\sqrt{T} \Phi_{l}\right\}_{l=0}^{L-1}$, where $L=2^{a}=2^{R_{m} T}$ which gives the code rate $R_{m}=\left(\log _{2} L\right) / T$ (bit/ channel use). Here, let $[\cdot]^{\dagger}$ denote real transpose matrix and $[\cdot]^{*}$ denote complex conjugate transpose matrix.

Suppose $\boldsymbol{X}_{l}$ is transmitted via the channel shown as Figure 1, then the $T \times N$ received signal matrix is:

$$
\boldsymbol{Y}=\sqrt{\frac{\rho}{M}} \boldsymbol{X}_{l} \boldsymbol{H}+\boldsymbol{W}=\sqrt{\frac{\rho T}{M}} \boldsymbol{\Phi}_{l} \boldsymbol{H}+\boldsymbol{W}
$$

where $\boldsymbol{H}$ is a $M \times N$ channel transfer matrix and $\boldsymbol{W}$ is a $T \times N$ additive white Gaussian noise (AWGN) matrix, both of whose entries obey $C N(0,1)$ distribution. The normalization factor $\sqrt{\rho T / M}$ of (1) ensures that the average signal noise ratio (SNR) at each receive antenna is $\rho$.

The receiver also contains two parts: noncoherent demodulator and belief-propagation (BP) decoder. $\boldsymbol{Y}$ is first demodulated as the soft information $\eta_{i}\left(i=0,1, \cdots, n_{c}-1\right)$ by the MAP demodulator and then $n_{c}$ soft information values $\eta_{0}, \eta_{1}, \cdots, \eta_{n_{c}-1}$ are fed into the BP decoder in parallel. The BP decoder outputs $\lambda_{i}$, $i=0,1, \cdots, n_{c}-1$ (or $\lambda_{j}, j=0,1, \cdots, k-1$ ) which are computed by means of the BP iterative decoding algorithm of the LDPC code. Finally, $k$ soft information values $\lambda_{0}, \lambda_{1}, \cdots, \lambda_{k-1}$ are fed to the decider and the de-

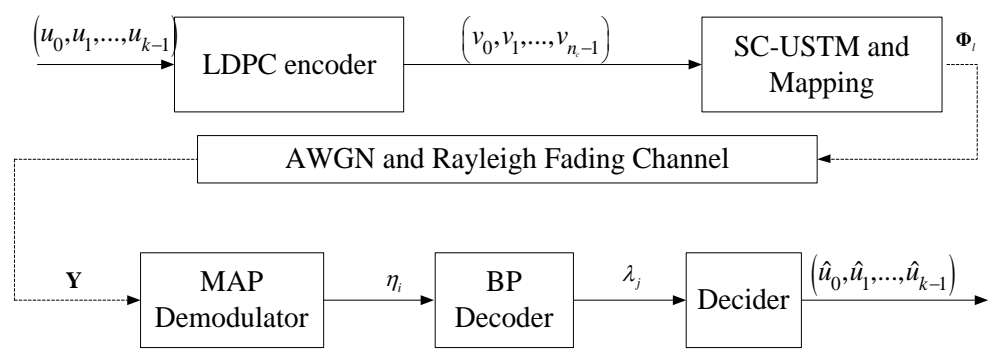

Figure 1. Noncoherent MIMO transmitter and receiver for the LDPC-coded SC-USTM. 
cider outputs the decoded information sequence $\left(\hat{u}_{0}, \hat{u}_{1}, \cdots, \hat{u}_{k-1}\right)$ in parallel. So far, a general encoding/modulating and demodulating/decoding noncoherent MIMO system without regard to channel estimation has been established. The spectral sufficiency of the MIMO system is $R=R_{c} R_{m}=k\left(\log _{2} L\right) / n_{c} T$ (bit/channel use).

In transmitter, the mapping from a CMS $\mathbf{z}_{l} \in\left\{\mathbf{z}_{l}\right\}_{l=0}^{L-1}$ to a TSM $\boldsymbol{X}_{l} \in\left\{\sqrt{T} \boldsymbol{\Phi}_{l}\right\}_{l=0}^{L-1}$ will be designed by considering synthetically the several factors, such as construction of transmit signal matrix, model of demodulating algorithm and model of distance measures.

We designed three MIMO systems according to different demodulating algorithm. The first system directly uses the MAP demodulating algorithm as demodulator in receiver and its transmitter adopts the natural mapping scheme. The so-called "natural mapping” means that $L$ binary CMSs $\left\{\mathbf{z}_{l}\right\}_{l=0}^{L-1}$ are ranked according to the natural order of decimal system which is a relationship of one-to-one correspondence with the natural order of the SC-USTM constellation $\left\{\sqrt{T} \boldsymbol{\Phi}_{l}\right\}_{l=0}^{L-1}=\left\{\sqrt{T}\left[\sin (\pi l / L) \cdot \boldsymbol{I}_{M} \quad \cos (\pi l / L) \cdot \boldsymbol{I}_{M}\right]_{T \times M}^{\dagger}\right\}_{l=0}^{L-1}$. The second system uses the presented dual demodulating scheme as demodulator in receiver and its mapping in transmitter is the Gray code of the set $\left\{\mathbf{z}_{l}\right\}_{l=0}^{L-1}$. This mapping scheme means that any two adjacent CMSs in this Gray code differ in only one bit and each of $L$ CMSs based on the rank of Gray code is still one to one match with the natural order of the SC-USTM constellation, i.e., $l=0,1, \cdots, L-1$. The hardware design and implementation of the Gray mapping, superficially at least, is more troublesome than that of the natural mapping. But late on, we will observe that the Gray mapping produces the benefit for reducing the complexity of dual demodulators by a large margin. The third system combines the presented dual demodulating scheme with the BP iterative decoding algorithm of the LDPC code to construct an iterative feedback system.

We claim to design a practical LDPC-coded SC-USTM system. Here the LDPC codes can be any available structure. In our simulation tests, the irregular QC-LDPC codes in IEEE 802.16e Standard [21] is selected as the outer code and the LDPC decoder uses the standard BP algorithm based on the bitwise reliability. The detail description of the LDPC encoder and decoder is omitted for brevity.

\section{The SC-USTM Map Demodulator}

In order to perform the decoding algorithm of the LDPC code, we need to get the LLR values of message bits from the demodulator of the SC-USTM, which should be analogous to the LLR of the observation from a BPSK demodulator over an AWGN channel. We do not repeat in detail the derivation of the general MAP demodulating algorithm based on USTM [7] [14] [15], but only derive the simplified MAP demodulating algorithm based on the structural SC-USTM first presented by [11] and then used by [19].

Let $\tau=0,1, \cdots, a-1$ denote the index of binary code bit in any of $L$ CMSs. We separate the set $\left\{\boldsymbol{\Phi}_{l}\right\}_{l=0}^{L-1}$ into two subsets $\Phi_{\tau}^{1}$ and $\Phi_{\tau}^{0}$, similarly the index $l=0,1, \cdots, L-1$ of the set $\left\{\mathbf{z}_{l}\right\}_{l=0}^{L-1}$ is also separated into two index subsets $l_{\tau}^{1}$ and $l_{\tau}^{0},\left|l_{\tau}^{1}\right|+\left|l_{\tau}^{0}\right|=L$, where $\Phi_{\tau}^{1}$ denotes the set of such matrix and $l_{\tau}^{1}$ of such index that the $\tau$ th bit of the CMS $\mathbf{z}_{l}$ is 1 and $\boldsymbol{\Phi}_{\tau}^{0}$ and $l_{\tau}^{0}$ respectively the set of such matrix or index that the $\tau$ th bit of $\mathbf{z}_{l}$ is 0 for $\tau=0,1, \cdots, a-1$. For any transmit signal matrix $\Phi_{l}$ and any receive signal matrix $\boldsymbol{Y}$, the LLR of the $\tau$ th bit in $\mathbf{z}_{l}$, i.e., the general MAP demodulating algorithm [14] [15] of USTM is given by

$$
\eta_{i}=\eta\left(z_{i}=z_{l, \tau}\right)=\log \frac{\operatorname{Pr}\left[z_{l, \tau}=1 \mid \boldsymbol{Y}\right]}{\operatorname{Pr}\left[z_{l, \tau}=0 \mid \boldsymbol{Y}\right]}=\log \frac{\sum_{\boldsymbol{\Phi}_{l} \in \boldsymbol{\Phi}_{\tau}^{1}} \exp \left(\operatorname{tr}\left\{\frac{1}{1+M / \rho T} \boldsymbol{Y}^{*} \boldsymbol{\Phi}_{l} \boldsymbol{\Phi}_{l}^{*} \boldsymbol{Y}\right\}\right)}{\sum_{\boldsymbol{\Phi}_{l} \in \boldsymbol{\Phi}_{\tau}^{0}} \exp \left(\operatorname{tr}\left\{\frac{1}{1+M / \rho T} \boldsymbol{Y}^{*} \boldsymbol{\Phi}_{l} \boldsymbol{\Phi}_{l}^{*} \boldsymbol{Y}\right\}\right)}
$$

where $z_{l, \tau}$ denotes the binary value of the $\tau$ th bit in the CMS $z_{l}$ and $z_{i}$ denotes the binary value of the $i$ th bit in codeword with length $n_{c}$, and both are one-to-one.

We find that (2) can be simplified from two directions. First, it contains the multiplication of four $T \times M$ $(M=N)$ complex matrices, and then, it requires all $L$ constellation point matrices to taking part in the calculation. We can simplify the multiplication of four complex matrices into addition and multiplication of several 
real numbers by using the structural feature of the SC-USTM. The further simplified scheme of reducing the number of matrices of (2) will be discussed in the next section.

According to the structural feature of the unitary space time matrix of SC-USTM, we can know that

$\boldsymbol{\Phi}_{l}=\left[\begin{array}{ll}\boldsymbol{S}_{M}^{\dagger} & \boldsymbol{C}_{M}^{\dagger}\end{array}\right]_{T \times M}^{\dagger}=\left[\begin{array}{lll}\sin (\pi l / L) \cdot \boldsymbol{I}_{M} & \cos (\pi l / L) \cdot \boldsymbol{I}_{M}\end{array}\right]^{\dagger}$ is a real matrix of satisfying $\boldsymbol{\Phi}_{l}^{\dagger} \boldsymbol{\Phi}_{l}=\boldsymbol{I}_{M}$, where $l=0,1, \cdots, L-1$ and $T=2 M$, and $M \geq 2$ and $N$ are any positive integer. For convenience of mathematical process, suppose the corresponding receive signal matrix has the form $\boldsymbol{Y}=\left[\begin{array}{ll}\boldsymbol{S}_{N}^{*} & \boldsymbol{C}_{N}^{*}\end{array}\right]^{*}$, where $\boldsymbol{S}_{N}$ and $\boldsymbol{C}_{N}$ are two $M \times N$ matrices in which all complex elements $a_{m n} \mathrm{e}^{\mathrm{i} \theta_{m n}}$ and $b_{m n} \mathrm{e}^{i \varphi_{m n}}$ denote the receive signals from $N$ receive antennas during $T=2 M$ for $m=0,1, \cdots, M-1$ and $n=0,1, \cdots, N-1$. Substituting $\Phi_{l}$ and $\boldsymbol{Y}$ into (2), by means of three types of triangle function transforms $2 \cos \phi \sin \phi=\sin 2 \phi$, $\sin ^{2} \phi=(1-\cos 2 \phi) / 2$ and $\cos ^{2} \phi=(1+\cos 2 \phi) / 2$, we get

$$
\begin{aligned}
& \operatorname{tr}\left(\boldsymbol{Y}^{*} \Phi_{l} \Phi_{l}^{\dagger} \boldsymbol{Y}\right) \\
& =\operatorname{tr}\left(\left[\begin{array}{ll}
\boldsymbol{S}_{N}^{*} & \boldsymbol{C}_{N}^{*}
\end{array}\right]\left[\begin{array}{c}
\sin (\pi l / L) \cdot \boldsymbol{I}_{M} \\
\cos (\pi l / L) \cdot \boldsymbol{I}_{M}
\end{array}\right]\left[\begin{array}{ll}
\sin (\pi l / L) \cdot \boldsymbol{I}_{M} & \cos (\pi l / L) \cdot \boldsymbol{I}_{M}
\end{array}\right]\left[\begin{array}{l}
\boldsymbol{S}_{N} \\
\boldsymbol{C}_{N}
\end{array}\right]\right) \\
& =\operatorname{tr}\left(\boldsymbol{S}_{N M}^{*} \boldsymbol{S}_{M N}\right) \sin ^{2}(\pi l / L)+\operatorname{tr}\left(\boldsymbol{C}_{N M}^{*} \boldsymbol{C}_{M N}\right) \cos ^{2}(\pi l / L) \\
& +\operatorname{tr}\left(\boldsymbol{S}_{N M}^{*} \boldsymbol{C}_{M N}+\boldsymbol{C}_{N M}^{*} \boldsymbol{S}_{M N}\right) \sin (\pi l / L) \cos (\pi l / L) \\
& =\frac{\operatorname{tr}\left(\boldsymbol{S}_{N M}^{*} \boldsymbol{S}_{M N}\right)+\operatorname{tr}\left(\boldsymbol{C}_{N M}^{*} \boldsymbol{C}_{M N}\right)}{2}+\frac{\operatorname{tr}\left(\boldsymbol{C}_{N M}^{*} \boldsymbol{C}_{M N}\right)-\operatorname{tr}\left(\boldsymbol{S}_{N M}^{*} \boldsymbol{S}_{M N}\right)}{2} \cos (2 \pi l / L) \\
& +\frac{\operatorname{tr}\left(\boldsymbol{S}_{N M}^{*} \boldsymbol{C}_{M N}+\boldsymbol{C}_{N M}^{*} \boldsymbol{S}_{M N}\right)}{2} \sin (2 \pi l / L) \\
& =\frac{\sum_{n=1}^{N} \sum_{m=1}^{M}\left(a_{m n}\right)^{2}+\sum_{n=1}^{N} \sum_{m=1}^{M}\left(b_{m n}\right)^{2}}{2}+\frac{\sum_{n=1}^{N} \sum_{m=1}^{M}\left(b_{m n}\right)^{2}-\sum_{n=1}^{N} \sum_{m=1}^{M}\left(a_{m n}\right)^{2}}{2} \cos (2 \pi l / L) \\
& +2 \sum_{n=1}^{N} \sum_{m=1}^{M} a_{m n} b_{m n} \cos \left(\theta_{m n}-\varphi_{m n}\right) \sin (2 \pi l / L) \\
& =\alpha+\beta \cos (2 \pi l / L)+2 \gamma \sin (2 \pi l / L)
\end{aligned}
$$

where

$$
\begin{gathered}
\alpha=\left(\sum_{n=1}^{N} \sum_{m=1}^{M}\left(b_{m n}\right)^{2}-\sum_{n=1}^{N} \sum_{m=1}^{M}\left(a_{m n}\right)^{2}\right) / 2, \\
\beta=\left(\sum_{n=1}^{N} \sum_{m=1}^{M}\left(b_{m n}\right)^{2}-\sum_{n=1}^{N} \sum_{m=1}^{M}\left(a_{m n}\right)^{2}\right) / 2, \\
\gamma=\sum_{n=1}^{N} \sum_{m=1}^{M} a_{m n} b_{m n} \cos \left(\theta_{m n}-\varphi_{m n}\right) .
\end{gathered}
$$

We rewrite the LLR of (2) as follows.

$$
\eta_{i}=\eta\left(z_{i}=z_{l, \tau}\right)=\log \frac{\sum_{l \in l_{t}^{1}, l_{\tau}^{1} \subset L} \exp \left(\frac{\alpha+\beta \cos (2 \pi l / L)+\gamma \sin (2 \pi l / L)}{1+M / \rho T}\right)}{\sum_{l \in l_{\tau}^{0}, l_{\tau}^{0} \subset L} \exp \left(\frac{\alpha+\beta \cos (2 \pi l / L)+\gamma \sin (2 \pi l / L)}{1+M / \rho T}\right)}
$$

We call the expression (3) the SC-USTM MAP demodulating algorithm.

\section{Dual-Demodulator of SC-USTM}

The drawback of the SC-USTM MAP demodulating algorithm of (3) is that all $L$ constellation point matrices 
take part in the calculation of (3), which results in the calculated amount of $L$ exponents. We create the dual demodulating scheme in order to decrease the exponent operations, or eliminate as many matrices taking part in the calculation of (3) as possible. The basic ideal is that the first demodulation uses the maximum likelihood (ML) algorithm of SC-USTM and the second demodulation uses the SC-USTM MAP algorithm presented in the above section, and Figure 2 gives the block diagram of the MIMO system based on the dual-demodulator.

\subsection{The SC-USTM ML Demodulating Algorithm}

It is necessary to explore the basic principle and the calculated complexity of the SC-USTM ML demodulating algorithm with a perspective different from [11]. For arbitrary positive integer $M \geq 2, N$ and $T=2 M$, under the condition of the transmit signal matrix being

$$
\boldsymbol{\Phi}_{l}=\left[\begin{array}{ll}
\boldsymbol{S}_{M}^{\dagger} & \boldsymbol{C}_{M}^{\dagger}
\end{array}\right]^{\dagger}=\left[\begin{array}{ll}
\sin (\pi l / L) \cdot \boldsymbol{I}_{M} & \cos (\pi l / L) \cdot \boldsymbol{I}_{M}
\end{array}\right]^{\dagger} \text {, we suppose that the } T \times N \text { receive signal matrix has }
$$

the form of $\boldsymbol{Y}=\left[\begin{array}{ll}\boldsymbol{S}_{N}^{*} & \boldsymbol{C}_{N}^{*}\end{array}\right]^{*}$, then the SC-USTM ML demodulating algorithm [19] [20] is

$$
\begin{aligned}
\boldsymbol{\Phi}_{M L} & =\arg \max _{\boldsymbol{\Phi}_{l} \in\left\{\boldsymbol{\Phi}_{l}\right\}_{l=0}^{L-1}} p\left(\boldsymbol{Y} / \boldsymbol{\Phi}_{l}\right) \\
& =\arg \max _{\boldsymbol{\Phi}_{l} \in\left\{\boldsymbol{\Phi}_{l}\right\}_{l=0}^{L-1}} \frac{\exp \left(-\operatorname{tr}\left\{\left[\boldsymbol{I}_{T}-\frac{1}{1+M / \rho T} \boldsymbol{\Phi}_{l} \boldsymbol{\Phi}_{l}^{\dagger}\right] \boldsymbol{Y} \boldsymbol{Y}^{\dagger}\right\}\right)}{\pi^{T N}(1+\rho T / M)^{M N}} \\
& =\arg \max _{\boldsymbol{\Phi}_{l} \in\left\{\boldsymbol{\Phi}_{\boldsymbol{l}} l_{l=0}^{L-1}\right.} \operatorname{tr}\left(\boldsymbol{Y}^{\dagger} \boldsymbol{\Phi}_{l} \boldsymbol{\Phi}_{l}^{\dagger} \boldsymbol{Y}\right) \\
& =\arg \max _{\boldsymbol{\Phi}_{l} \in\left\{\boldsymbol{\Phi}_{\boldsymbol{l}} l_{l=0}^{L-1}\right.}(\alpha+\beta \cos (2 \pi l / L)+2 \gamma \sin (2 \pi l / L)) \\
& =\arg \max _{\boldsymbol{\Phi}_{l} \in\left\{\boldsymbol{\Phi}_{l} l_{l=0}^{L-1}\right.}(\alpha+\xi \cos (2 \pi l / L-\delta))
\end{aligned}
$$

where

$$
\begin{gathered}
\xi=\sqrt{\beta^{2}+(2 \gamma)^{2}} \\
=\sqrt{\frac{\left(\sum_{n=1}^{N} \sum_{m=1}^{M}\left(b_{m n}\right)^{2}-\sum_{n=1}^{N} \sum_{m=1}^{M}\left(a_{m n}\right)^{2}\right)^{2}}{4}+4\left(\sum_{n=1}^{N} \sum_{m=1}^{M} a_{m n} b_{m n} \cos \left(\theta_{m n}-\varphi_{m n}\right)\right)^{2}}, \\
\delta=\operatorname{arctg} \frac{2 \gamma}{\beta}=\operatorname{arctg} \frac{4 \sum_{n=1}^{N} \sum_{m=1}^{M} a_{m n} b_{m n} \cos \left(\theta_{m n}-\varphi_{m n}\right)}{\sum_{n=1}^{N} \sum_{m=1}^{M}\left(b_{m n}\right)^{2}-\sum_{n=1}^{N} \sum_{m=1}^{M}\left(a_{m n}\right)^{2}}
\end{gathered}
$$

and $p\left(\boldsymbol{Y} / \Phi_{l}\right)$ is the conditional probability of $\boldsymbol{Y}$ given $\boldsymbol{\Phi}_{l}$.

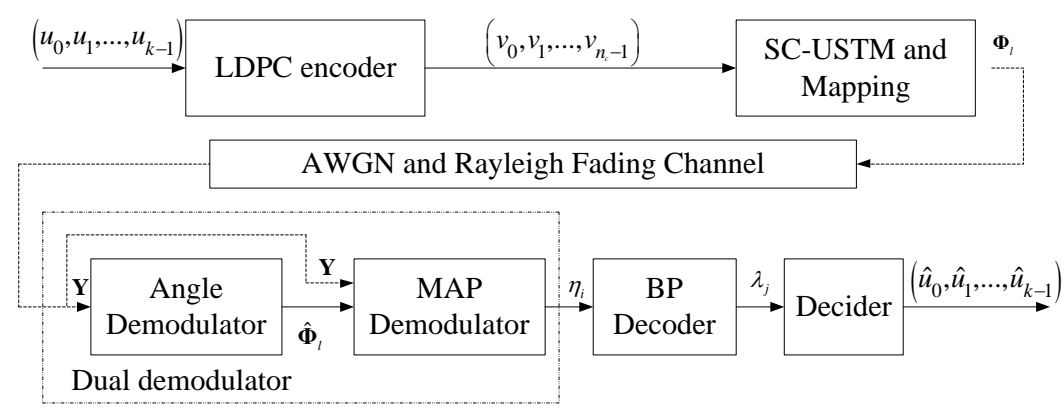

Figure 2. Noncoherent MIMO transmitter and receiver for the LDPC-coded SCUSTM system equipped with the dual-demodulator. 
If making $\cos (2 \pi l / L-\delta)=1$ for (4), then the likelihood probability $p\left(\mathbf{Y} / \boldsymbol{\Phi}_{l}\right)$ can achieve the maximum value. From relationship $\cos (2 \pi l / L-\delta)=1$, we can solve the following sequence number of constellation points by plugging (5) into (4):

$$
\begin{aligned}
& l=\lfloor|\delta L / 2 \pi+0.5|\rfloor(\bmod L) \\
& =\left\lfloor\frac{L}{2 \pi} \operatorname{arctg} \frac{4 \sum_{n=1}^{N} \sum_{m=1}^{M} a_{m n} b_{m n} \cos \left(\theta_{m n}-\varphi_{m n}\right)}{\sum_{n=1}^{N} \sum_{m=1}^{M}\left(b_{m n}\right)^{2}-\sum_{n=1}^{N} \sum_{m=1}^{M}\left(a_{m n}\right)^{2}}+0.5 \|(\bmod L)\right.
\end{aligned}
$$

where $\lfloor x\rfloor$ denotes the largest integer that does not exceed $x, \bmod L$ denotes that $l$ is a positive integer and its range is $0 \leq l \leq L-1$. The function of taking the floor $\lfloor x\rfloor$ may introduce the following error. If the fractional part of $\lfloor|\delta L / 2 \pi|\rfloor$ is less than 0.5 , then the demodulating error of $\lfloor|\delta L / 2 \pi|\rfloor=l$ is small, or $l$ is the correct sequence number of the demodulated signal matrix; if the fractional part of $[\delta \delta / 2 \pi \mid\rfloor$ is larger than 0.5 , then the demodulating error of $\lfloor|\delta L / 2 \pi|\rfloor=l$ is large. For relieving the error, we make $[|\delta L / 2 \pi+0.5|\rfloor$ replace $\lfloor|\delta L / 2 \pi|\rfloor$. Let $l$ be the demodulated sequence number. If the fractional part of $\lfloor|\delta L / 2 \pi|\rfloor$ is less than 0.5 , then $\lfloor|\delta L / 2 \pi+0.5|\rfloor=l$; if the fractional part of $\lfloor|\delta L / 2 \pi|\rfloor$ is larger than 0.5 , then

$\lfloor|\delta L / 2 \pi+0.5|\rfloor=l+1$. We call (6) the SC-UATM ML demodulating algorithm, or the angle demodulator for short.

Here, we give the complexity analysis of the angle demodulator. From (6), we can know that

$a_{m n}, b_{m n}, \theta_{m n}, \varphi_{m n}$ are the known parameters provided by all complex elements in receive signal matrix. Once the values of $L, M, N$ are given, the calculating amount of (6) is $4 M N+2$ real additions and $4 M N+4$ real multiplications, plus one arc tangent. The SC-UATM ML demodulating algorithm is independent of the size of constellation $L$, the coherent time $T$ and the date rate $R_{m}$ and its complexity is very low and a linear relationship with $M$ and $N$, i.e., the demodulating complexity is $O(M N)$.

\subsection{The Dual Demodulator of SC-USTM}

We observe the MAP demodulating algorithm of the SC-USTM in (3) and discover that the summing expression in numerator and denominator requires all $L$ constellation points to take part in the calculation of (3). For reducing the number of constellation points of taking part in the calculation of (3), we create the dual demodulator which is wished to decrease the calculated quantity from $L$ points to $\hat{L}<L$ points, where $\hat{L}$ denotes the number of those constellation points of taking part in the calculation of (3) after reducing $L$. Let $\{\hat{L}\}$ denote the set of index values of $\hat{L}$ signal matrices.

Our basic thought is: firstly, demodulate a proximate signal matrix $\hat{\boldsymbol{\Phi}}_{1}$ by exploiting the angle demodulator of the SC-USTM; then make the demodulated $\hat{\boldsymbol{\Phi}}_{l}$ (or $l$ ) as the central point and select three points, five points, seven points and so on, i.e., $l, l \pm 1, l \pm 2, \cdots \in\{\hat{L}\}$ which are viewed as those points of taking part in calculation of (3) until they achieve some acceptable performance.

The angle demodulator of (6) may lead to the error toward two directions. This is that the $l$ th transmit signal matrix is more likely to be improperly demodulated as the $l-1$ th matrix or the $l+1$ th matrix. According to this feature, we choose the Gray code to design the coded mapping sequence. This mapping scheme that the sequence number $l=0,1, \cdots, L-1$ of transmit signal matrix has a one-to-one correspondence with the codeword of the coded mapping sequence based on Gray code can make the demodulating error achieve a degree as small as possible.

Let $\hat{L}=3,5,7$ points which respectively corresponds to three cases: $\hat{L}=3$ for $L=16, \hat{L}=5$ for $L=32$, $\hat{L}=7$ for $L \geq 64$. When $l_{\tau}^{1}, l_{\tau}^{0} \subseteq\{\hat{L}\}$ and $l_{\tau}^{1}+l_{\tau}^{0}=\hat{L}$, then (3) can be modified as follows

$$
\eta_{i}=\log \frac{\sum_{l \in l_{\tau}^{1}, l_{\tau}^{1} \subseteq\{\hat{L}\}} \exp \left(\frac{\alpha+\beta \cos (2 \pi l / L)+\gamma \sin (2 \pi l / L)}{1+M / \rho T}\right)}{\sum_{l \in l_{\tau}^{0}, l_{\tau}^{0} \subseteq\{\hat{L}\}} \exp \left(\frac{\alpha+\beta \cos (2 \pi l / L)+\gamma \sin (2 \pi l / L)}{1+M / \rho T}\right)}
$$

We call (7) the simplified MAP demodulating algorithm of the SC-USTM. 
We did the simulation experiments for three demodulating algorithms, i.e., the angle demodulator of (6), the SC-USTM MAP demodulating algorithm of (3) and the simplified MAP demodulating algorithm of (7) combining with the dual demodulator. Figure 3 gives the comparison results of their performance curves. The experiment conditions are setup as follows. The structural parameters of the SC-USTM are $M=N=2, T=2 M=4$, and the size of constellation is $L=16$. The structural parameters of the QC-LDPC codes from IEEE 802.16e Standard are code rate $R_{c}=1 / 2$ and code length $n_{c}=2304$.

In Figure 3, the dash line shows the performance of the angle demodulator without the LDPC code, this is that the MIMO system with the SC-USTM and its angle demodulator of (6) operates in high-SNR region which is $28 \mathrm{~dB}$ at a BER of $2.5 \times 10^{-6}$ and at the data rate which is $R=R_{m}=\left(\log _{2} L\right) T=1$ (bit/ channel use).

The dot line with the square symbol shows the performance of the LDPC coded SC-USTM MIMO system of combining the SC-USTM MAP demodulating algorithm of (3) with the BP decoder at the receiver, and the LDPC coded SC-USTM MIMO system improves the performance about $15 \mathrm{~dB}$ at the BER level of $1.0 \times 10^{-4}$, compared to the performance of the uncoded SC-USTM MIMO system, and the data rate of this MIMO system is $R=R_{m} R_{c}=k \times\left(\log _{2} L\right) / n_{c} \times T=0.5$ (bit/ channel use).

The solid line with circle symbol shows the performance of the LDPC coded SC-USTM MIMO system with the dual demodulator. This scheme needs only three points $\hat{L}=3$ take part in the calculation of (7) and improves the performance about $17.0 \mathrm{~dB}$ at the BER level of $1.0 \times 10^{-6}$, compared to the performance of the uncoded SC-USTM MIMO system, but deteriorates the performance about $1.0 \mathrm{~dB}$ at the BER level of $1.0 \times 10^{-4}$, compared to the performance of the SC-USTM MAP demodulating algorithm with $L=16$ points of (3).

Therefore, the simplified MAP of (7) and the general MAP of (3) for the SC-USTM demodulating algorithm set forth a tradeoff between the performance and the complexity for the LDPC coded SC-USTM MIMO system.

\section{Dual-Demodulate-Decoder with Iterative Feedback}

\subsection{Iterative Dual-Demodulate-Decoder}

In order to improve the performance, the dual-demodulator in Figure 2 can be modified into the dual-demodulate-decoder with iterative feedback loop as shown in Figure 4. The key problem is how to design the interface from the decoder output port to the MAP demodulator input port. The BP decoder outputs $n_{c}$ soft values $\lambda_{i}\left(i=0,1, \cdots, n_{c}-1\right)$ in parallel. On the one hand, $k$ effective soft information values $\lambda_{j}(j=0,1, \cdots, k-1)$ taken from $n_{c}$ soft values $\lambda_{i}\left(i=0,1, \cdots, n_{c}-1\right)$ are fed into the decider which gives the decoded information sequence $\left(\hat{u}_{0}, \hat{u}_{1}, \cdots, \hat{u}_{k-1}\right)$. On the other hand, $n_{c}$ soft values $\lambda_{i}$ can be delivered to the input port of the MAP demodulator by a feedback loop. Note that $\lambda_{i}$ cannot directly be fed into the MAP demodulator, because the MAP demodulator needs the input of the priori probability of the $i$ th bit $z_{i}=z_{l, \tau}$ of the codeword with the

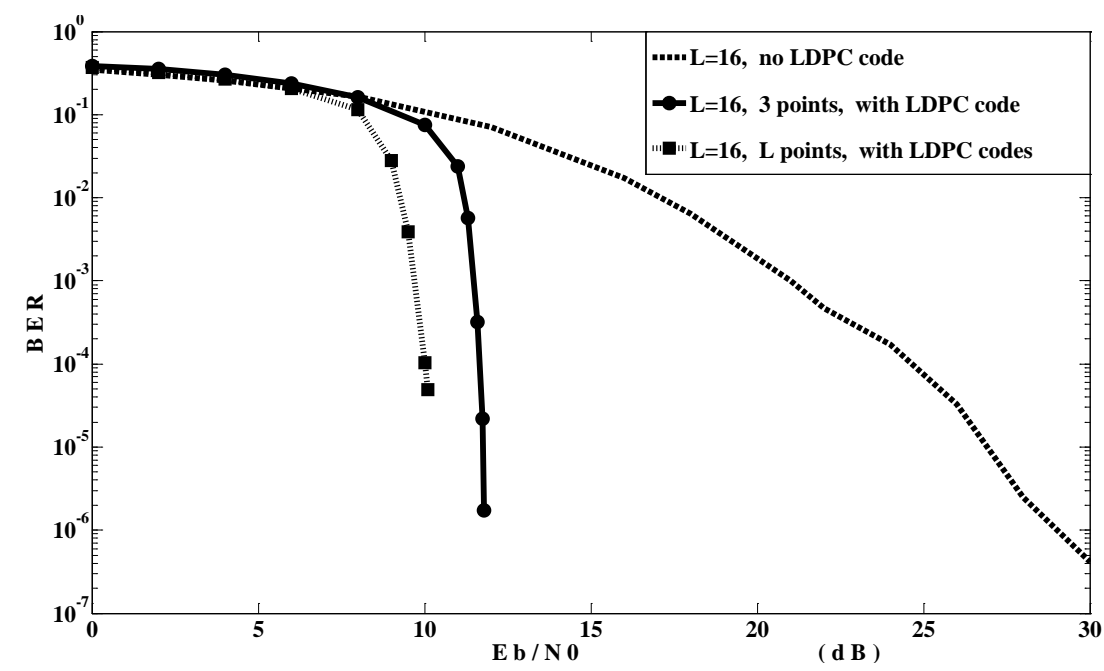

Figure 3. Performance curves for the 3 and 16 points dual-demodulate-decoders of the LDPC-coded SC-USTM system, as well as for the angle demodulator without LDPC code. 


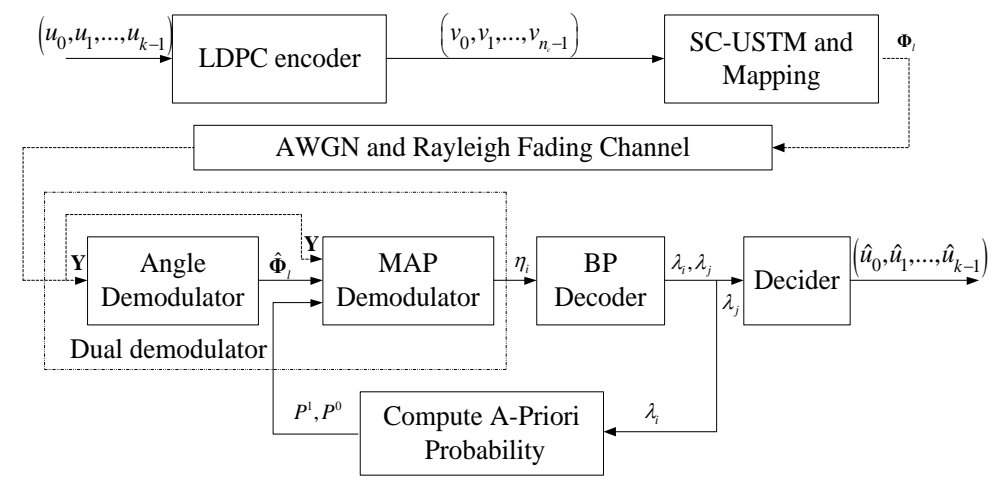

Figure 4. Noncoherent MIMO transmitter and receiver for the LDPC-coded SC-USTM system equipped with the iterative dual-demodulator.

size $n_{c}$. Therefore, in the feedback loop, the well-known priori probability $P^{1}=P\left(z_{i}=z_{l, \tau}=1\right)$ and $P^{0}=P\left(z_{i}=z_{l, \tau}=0\right)$ of $z_{i}=z_{l, \tau}$ are computed as follows:

$$
P^{1}=\frac{\mathrm{e}^{\lambda_{i}}}{\mathrm{e}^{\lambda_{i}}+1}, \quad P^{0}=\frac{1}{\mathrm{e}^{\lambda_{i}}+1}
$$

So (7) can be modified as:

$$
\begin{aligned}
& \eta_{i}=\eta_{\text {loop }}\left(z_{i}=z_{l, \tau}\right)=\log \frac{\sum_{l \in l_{\tau}^{1}, l_{\tau}^{1} \subseteq\{\hat{L}\}} \exp \left(\frac{\alpha+\beta \cos (2 \pi l / L)+\gamma \sin (2 \pi l / L)}{1+M / \rho T}\right) \frac{\mathrm{e}^{\lambda_{i}}}{\mathrm{e}^{\lambda_{i}}+1}}{\sum_{l \in l_{\tau}^{0}, l_{\tau}^{0} \subseteq\{\hat{L}\}} \exp \left(\frac{\alpha+\beta \cos (2 \pi l / L)+\gamma \sin (2 \pi l / L)}{1+M / \rho T}\right) \frac{1}{\mathrm{e}^{\lambda_{i}}+1}} \\
& =\log \frac{\sum_{l \in l_{\tau}^{1}, l_{\tau}^{1} \subseteq\{\{\hat{L}\}} \exp \left(\frac{\alpha+\beta \cos (2 \pi l / L)+\gamma \sin (2 \pi l / L)}{1+M / \rho T}\right)}{\sum_{l \in l_{\tau}^{0}, l_{\tau}^{0} \subseteq\{\{\hat{L}\}} \exp \left(\frac{\alpha+\beta \cos (2 \pi l / L)+\gamma \sin (2 \pi l / L)}{1+M / \rho T}\right)}+\lambda_{i}
\end{aligned}
$$

We call (9) the iterative dual-demodulating algorithm of the SC-USTM.

\subsection{Improving Scheme of Iterative Dual-Demodulating Algorithm}

We observe the values of $\left(\lambda_{0}, \lambda_{1}, \cdots, \lambda_{n_{c}-1}\right)$ outputted from the BP decoder and find that the value range of $\lambda_{i}$ is between 0 and 1 . Therefore, the boundary of decision in decider is set as 0.5 , i.e., when $\lambda_{i}>0.5$, the decoded bit $\hat{u}_{i}=1$; when $\lambda_{i}<0.5, \hat{u}_{i}=0$. If we modify each $\lambda_{i}$ by means of translating $\lambda_{i}$ into $\hat{\lambda}_{i}=\lambda_{i}-0.5$, then we can obtain the following decision: when $\hat{\lambda}_{i}=\lambda_{i}-0.5>0, \hat{u}_{i}=1$; when $\hat{\lambda}_{i}=\lambda_{i}-0.5<0, \hat{u}_{i}=0$. The theoretical basis of this modification is that comparison of numerical magnitudes, such as $\lambda_{i} \gtreqless 0.5$, is changed into the detection of plus or minus, or a zero-crossing detector is more accurate and has lower complexity than a one-nonzero-threshold detector in decision device. This modification makes the priori probability values of (8) change as follows

$$
P^{1}=\frac{\mathrm{e}^{\lambda_{i}-05}}{\mathrm{e}^{\lambda_{i}-0.5}+1}, \quad P^{0}=\frac{1}{\mathrm{e}^{\lambda_{i}-0.5}+1}
$$

and the iterative dual-demodulating algorithm of (9) is changed as follows

$$
\eta_{i}=\eta_{\text {loop }}\left(z_{i}=z_{l, \tau}\right)=\log \frac{\sum_{l \in l_{\tau}^{l}, l_{\tau}^{1} \leq\{\hat{L}\}} \exp \left(\frac{\alpha+\beta \cos (2 \pi l / L)+\gamma \sin (2 \pi l / L)}{1+M / \rho T}\right)}{\sum_{l \in l_{t}^{0}, l_{\tau}^{0} \leq\{\hat{L}\}} \exp \left(\frac{\alpha+\beta \cos (2 \pi l / L)+\gamma \sin (2 \pi l / L)}{1+M / \rho T}\right)}+\lambda_{i}-0.5
$$


We call (11) the improved iterative dual-demodulating algorithm of the SC-USTM.

Figure 5 shows the performance comparison of four schemes. The dot line and the solid line with circle symbol respectively denote the performance curves of the SC-USTM MAP demodulating algorithm of (3) and the simplified SC-USTM MAP demodulating algorithm with three points of (7), which is the same as Figure 3.

The solid line with square symbol shows the performance curve of the dual demodulator with three points and one iterative feedback for (9), which is superior to the performance of the simplified SC-USTM MAP demodulating algorithm of (7) about $0.8 \mathrm{~dB}$ at the BER level of $1.0 \times 10^{-6}$.

The solid line with rhombus symbol shows the performance curve of improved iterative dual-demodulating algorithm of (11). The complexity from (9) to (11) does not almost increase, but the performance improves 0.5 $\mathrm{dB}$ at $10^{-6}$ BER.

Note that when the iterative time of feedback loop in Figure 4 is larger than 2, the performance improvement is unremarkable but the computational complexity of the demodulator increases by multiples, the reason will be discussed late on.

\section{Complexity Analysis}

In this section, we roughly assess the computational complexity of several demodulating schemes of the different noncoherent space time receivers by comparing the presented LDPC coded SC-USTM noncoherent demodulator with the existing turbo coded noncoherent space-time demodulator based on information-bearing pilots and spatial multiplexing in [7]. We first give the computational complexities of several demodulating algorithms, and then compare them with the complexity of the existing noncoherent space time demodulators.

The total calculated amount of (3) is one complex logarithm, $L$ complex exponents, $(6 M N+4) \times L$ real additions, $(6 M N+5) \times L$ real multiplications and $(M N+2) L$ trigonometric calculations.

The total calculated amount of (2) is one complex logarithm, $L$ complex exponents, $M N T L(3 T-5) / 2$ real additions and $\operatorname{MNTL}(4 T-1)$ real multiplications and $\operatorname{MNTL}(T-1) / 2$ trigonometric calculations.

The well-known coherent MAP demodulating algorithm of (10) in [19] needs one complex logarithm, $L$ complex exponents, $(4 N+2) M L$ real additions and $(4 N+4) M L$ real multiplications.

For example, let $T=2 M=2 N=4$ and $L=16$, except the same part of complex logarithm and exponents, for the rest part containing real addition and multiplication, the complexity of the SC-USTM MAP demodulating algorithm using (3) is small 4.5 times on average, compared to the complexity of the general MAP demodulating algorithm using (2), and is about 1.3 times larger than the complexity of the coherent MAP demodulating algorithm in [19].

Except for the same calculation of one logarithm and $L$ exponents, the difference of the calculated amount between (2) and (3) is mainly resulted from $\operatorname{tr}\left(\boldsymbol{Y}^{*} \boldsymbol{\Phi}_{l} \boldsymbol{\Phi}_{l}^{*} \boldsymbol{Y}\right)$. In (2), the transmit signal matrix $\boldsymbol{\Phi}_{l}$ taking parting

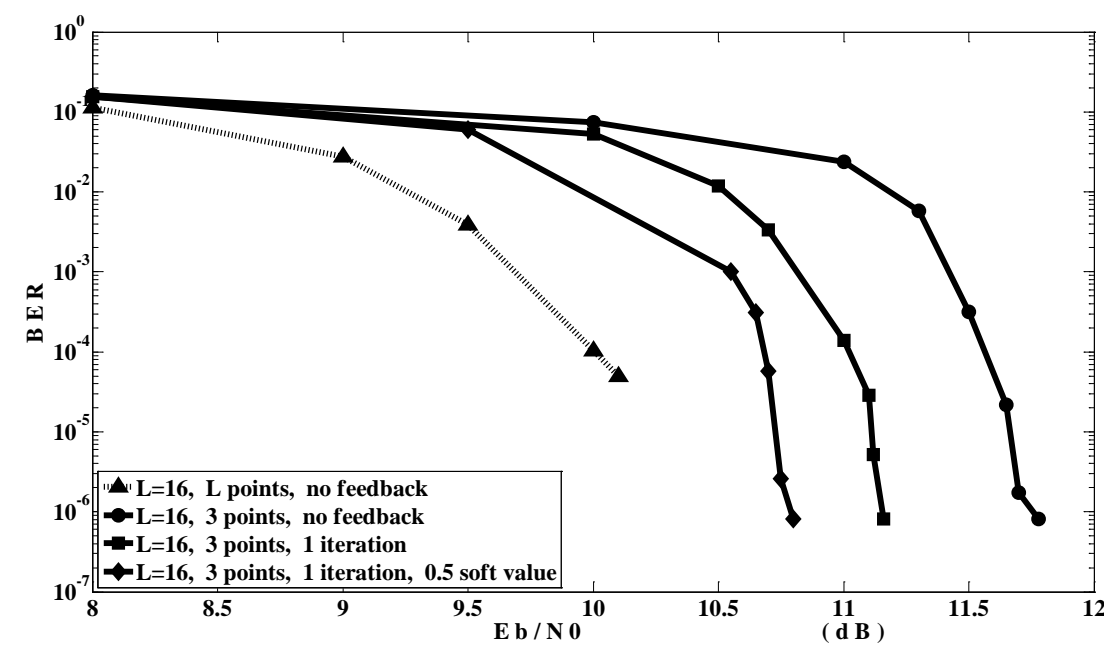

Figure 5. Performance curves for four dual-demodulators, 3 and 16 points, one iteration and modifying parameter $\lambda_{i}$. 
in the calculation of $\operatorname{tr}\left(\boldsymbol{Y}^{*} \Phi_{l} \Phi_{l}^{*} \boldsymbol{Y}\right)$ is a complex matrix in which all elements are the modulation symbol of MQAM or M-PSK, but in (3), $\boldsymbol{\Phi}_{l}$ is a sparse real matrix consisted of zero elements and real elements of trigonometric function which results in the simplified calculation of $\operatorname{tr}\left(\boldsymbol{Y}^{*} \boldsymbol{\Phi}_{l} \boldsymbol{\Phi}_{l}^{*} \boldsymbol{Y}\right)$. The difference of the calculated amount between (3) in this paper and (10) in [19] is that the noncoherent USTM demodulation, for example (3), is performed by considering all signal matrix $\boldsymbol{\Phi}_{l}$, but the coherent demodulation, for example (10) in [19], by only modulation symbol-wise.

As the calculated amount of the angle demodulating algorithm of (6) is $4 M N+2$ real additions and $4 M N+4$ real multiplications plus one arc tangent, so the total calculated amount of the simplified SC-USTM MAP demodulating algorithm with $\hat{L}$ points of (7) is one complex logarithm, $\hat{L}$ complex exponents,

$M N T \hat{L}(3 T-5) / 2$ real additions and $\operatorname{MNT\hat {L}}(4 T-1)$ real multiplications and $M N T \hat{L}(T-1) / 2$ trigonometric calculations for $\hat{L}=3,5,7$.

In order to determine the total calculated amount of the simplified MAP demodulating algorithm of (10) in [7] based on the orthogonal USTM presented in [12], we need to know the calculated amount of $\operatorname{tr}\left(\boldsymbol{Y}^{*} \boldsymbol{\Phi}_{l} \boldsymbol{\Phi}_{l}^{*} \boldsymbol{Y}\right)$. It is $M N T(3 T-5) / 2$ real additions, $M N T(4 T-1)$ real multiplications and $M N T(T-1) / 2$ trigonometric calculations. So (10) in [7] needs about twice calculated amount of $\operatorname{tr}\left(\boldsymbol{Y}^{*} \boldsymbol{\Phi}_{l} \boldsymbol{\Phi}_{l}^{*} \boldsymbol{Y}\right)$ and its complexity is about $O\left(2 M N T^{2}\right)$. The complexity of the above two demodulating algorithms is independent on the size of constellation $L$, and they exploit the special structural feature of the transmit signal matrix to achieve the goal of reducing the calculated amount of demodulating algorithm. Obviously, the calculated amount of the angle demodulating algorithm of (6) is less at least $T$ times than that of the simplified MAP demodulating algorithm of (10) in [7].

We design three LDPC coded SC-USTM noncoherent MIMO receivers without estimating the CSI which completely differ from the existing coded space-time noncoherent receivers in [7]. If the LDPC codes as outer code in these three receivers have the same construction and their decoders use the same BP decoding algorithm with 20 iterations, which means to have the same decoding complexity, then the main difference of three receivers rests with the distinct demodulators. Therefore, we need only to analyze the complexity of the whole demodulating scheme of these receivers to the exclusion of LDPC decoder. The first receiver is equipped by the SC-USTM MAP demodulator of (2) whose complexity is the total calculated amount of (3), i.e., $O(6 M N L)$, see the Subsection $B$ of the Section III. The second receiver is mounted by the dual-demodulator, consisting of the angle demodulator and the simplified SC-USTM MAP demodulator with three points, whose complexity is the sum of the total calculated amount of (6) and (7), i.e. $O\left(M N T^{2} \hat{L}\right)$. When $M, N$ are given and $T=2 M$ (the structural constraint of SC-USTM satisfying $\cos ^{2} x+\sin ^{2} x=1$ ) is fixed, then the second receiver has lower complexity than the first receiver as the size of constellation $L$ increases. The third receiver consists of one iterative feedback dual-demodulator/decoder whose complexity is twice sum of the total calculated amount of (6) and (7) as well as repeat iterations of decoder. When $L$ is very large, the complexity of the third receiver is lower than that of the first receiver.

A Turbo coded noncoherent space-time modulation using information-bearing pilots and spatial multiplexing (SM) is presented in [7], and in its receiver, called as iterative detection and decoding (IDD) receiver, demodulating scheme consists of two parts. The first part consists of a simplified noncoherent MAP demodulating algorithm of (10) in [7] and the second part has a more complex structure which consists of three modules such as channel estimator, coherent demodulator and signal matrix detector. The calculated amount of channel estimator is determined by a preliminary LMMSE channel estimate $\hat{\boldsymbol{H}}=\sqrt{\frac{M}{\rho}}\left(\frac{M}{\rho} \boldsymbol{I}_{M}+\boldsymbol{\Phi}_{l} \boldsymbol{\Phi}_{l}^{*}\right)^{-1} \boldsymbol{\Phi}_{l}^{*} \boldsymbol{Y}$ which contains matrix inversion and multiplication of three matrices which is not less than the calculated amount of multiplication of four matrices. The calculated amount of signal matrix detector is determined by (11) in [7] which involves in matrix inversion and multiplication of five matrices, as well as traversal calculation of $2^{\delta}$ candidate points. Obviously, the calculated amount of channel estimator is larger than that of (7). Thus it can be concluded that the complexity of the second and third receiver is lower than the IDD receiver in [7].

The special construction of the SC-USTM makes it emerge two extreme cases that on the one hand, it can generate by itself the lowest complexity demodulating algorithm based on ML and MAP; on the other hand, its independent running performance is worst, among all existing USTMs of [10]-[13]. The main reason is explained as follows. The distance feature of the SC-USTM is inferior to that of orthogonal USTM. Therefore, it is not possible to have the better performance, and this is reason what we have not tried our best to find the way to 
improve performance. The SC-USTM has such structural feature that the matrix elements contains zero elements as many as possible and special type similar to standard function, such as trigonometric function, which is the determining factor of resulting in the simplified demodulating algorithm.

\section{Conclusion and Natural Works}

We have designed three low complexity receivers for the LDPC-coded USTM noncoherent MIMO system which need not send the pilot information at the transmitter and estimate the channel state information at the receiver. The computational complexity of the second and third receivers is independent on the size of constellation and the coherent interval. Therefore, they are lower than that of the existing noncoherent space-time receivers.

\section{Funding}

This work is supported by National Natural Science Foundation of China under Grant No. 61071069.

\section{References}

[1] Telatar, E. (1999) Capacity of Multi-Antenna Gaussian Channels. European Transactions on Telecommunications, 10, 585-595. http://dx.doi.org/10.1002/ett.4460100604

[2] Foschini, G.J. (1996) Layered Space-Time Architecture for Wireless Communication in a Fading Environment When Using Multi-Element Antennas. Bell System Technical Journal, 1, 41-59. http://dx.doi.org/10.1002/bltj.2015

[3] Marzetta, T.L. and Hochwald, B. (1999) Capacity of a Mobile Multiple-Antenna Communication Link in Rayleigh-Flat Fading. IEEE Transactions on Information Theory, 45, 139-157. http://dx.doi.org/10.1109/18.746779

[4] Hochwald, B.M. and Marzetta, T.L. (2000) Unitary Space-Time Modulation for Multiple-Antenna Communications in Rayleigh Flat Fading. IEEE Transactions on Information Theory, 46, 543-564. http://dx.doi.org/10.1109/18.825818

[5] Zheng, L. and Tse, D.N.C. (2002) Communication on the Grassmann Manifold: A Geometric Approach to the Noncoherent Multiple-Antenna Channel. IEEE Transactions on Information Theory, 48, 359-383. http://dx.doi.org/10.1109/18.978730

[6] Krishnamoorthy, A. and Anastasopoulos, A. (2005) Code and Receiver Design for the Noncoherent Fast-Fading Channel. IEEE Journal on Selected Areas in Communications, 23, 1769-1778. http://dx.doi.org/10.1109/JSAC.2005.853802

[7] Chen Y. and Ueng, Y. (2011) Turbo Coded Noncoherent Space-Time Modulation Using Information-Bearing Pilots and Spatial Multiplexing. IEEE Transactions on Communications, 59, 1543-1559. http://dx.doi.org/10.1109/TCOMM.2011.042111.100169

[8] Hassibi, B. and Hochwald, B.M. (2003) How Much Training Is Needed in Multiple-Antenna Wireless Links? IEEE Transactions on Information Theory, 49, 951-963. http://dx.doi.org/10.1109/TIT.2003.809594

[9] Yu, Y., Giannakis, G.B. and Jindal, N. (2007) Information-Bearing Noncoherently Modulated Pilots for MIMO Training. IEEE Transactions on Information Theory, 53, 1160-1168. http://dx.doi.org/10.1109/TIT.2006.890693

[10] Hochwald, B.M., Marzetta, T.L., Richardson, T.J., Sweldens, W. and Urbanke, R. (2000) Systematic Design of Unitary Space-Time Constellations. IEEE Transactions on Information Theory, 46, 1962-1973. http://dx.doi.org/10.1109/18.868472

[11] Tarokh, V. and Kim, I.M. (2002) Existence and Construction of Noncoherent Unitary Space-Time Codes. IEEE Transactions on Information Theory, 48, 3112-3117. http://dx.doi.org/10.1109/TIT.2002.805075

[12] Zhao, W., Leus, G. and Gannakis, G.B. (2004) Orthogonal Design of Unitary Constellations for Uncoded and Trellis-Coded Noncoherent Space-Time Systems. IEEE Transactions on Information Theory, 50, 1319-1327. http://dx.doi.org/10.1109/TIT.2004.828154

[13] Gohary, R.H. and Davidson, T.N. (2009) Noncoherent MIMO Communication: Grassmannian Constellations and Efficient Detection. IEEE Transactions on Information Theory, 55, 1176-1205. http://dx.doi.org/10.1109/TIT.2008.2011512

[14] Bahceci, I. and Duman, T.M. (2002) Combined Turbo Coding and Unitary Space-Time Modulation. IEEE Transactions on Communications, 50, 1244-1249. http://dx.doi.org/10.1109/TCOMM.2002.801484

[15] Wei, R., Hsu, Y. and Pan, C. (2009) A Low-Complexity Noncoherent Iterative Space-Time Demodulator. IEEE Transactions on Communications, 57, 2895-2898. http://dx.doi.org/10.1109/TCOMM.2009.10.080056

[16] Sun, Z. and Tjhung, T.T. (2004) Multiple-Trellis-Coded Unitary Space-Time Modulation in Rayleigh Flat Fading. 
IEEE Transactions on Wireless Communications, 3, 2335-2344.

[17] Tran, N.H., Nguyen, H.H. and Tho, L.N. (2007) Coded Unitary Space-Time Modulation with Iterative Decoding: Error Performance and Mapping Design. IEEE Transactions on Communications, 55, 703-716.

[18] Vu, H.G., Nguyen, H.H. and Dodds, D.E. (2008) Performance Bound for LDPC Coded Unitary Space-Time Modulation. Wireless Personal Communications, 47, 383-397. http://dx.doi.org/10.1007/s11277-008-9487-0

[19] Stefanov, A. and Duman, T.M. (2001) Turbo-Coded Modulation for Systems with Transmit and Receive Antenna Diversity over Block Fading Channels: System Model, Decoding Approaches, and Practical Considerations. IEEE Journal on Selected Areas in Communications, 19, 958-968. http://dx.doi.org/10.1109/49.924879

[20] Peng, L., Yang, L. and Peng, Q. (2011) A Low-Complexity IRA-LDPC Coded Noncoherent Unitary Space-Time Modulation System on Rayleigh Flat Fading Channel. 2011 2nd International Conference on Mechanic Automation and Control Engineering, Hohhot, 15-17 July 2011, 7115-7118.

[21] (2005) IEEE P802.16e, D12: Draft IEEE Standard for Local Metropolitan Area Networks. Part 16: Air Interface for Fixed and Mobile Broadband Wireless Access Systems. 